\title{
ON DIFFERENTIABLE FUNCTIONS
}

\author{
BY FU CHENG HSIANG
}

Communicated by S. Bochner, May 25, 1960

1. Let $f(x)$ be a real function defined over the closed interval $[a, b]$ and differentiable at each point of the interval. The following is a well-known classical theorem.

Darboux's Theorem. If $\xi, \eta \in[a, b]$ and $f^{\prime}(\xi)=c, f^{\prime}(\eta)=d$ and $c<d$, then, if $c<k<d$, there exists at least one point $\zeta$ lying between $\xi$ and $\eta$ such that $f^{\prime}(\zeta)=k$.

Recently in 1947, Clarkson [1] developed the above property into the following important theorem concerning the behavior of the derivative $f^{\prime}(x)$.

Clarkson's Theorem. Let $\alpha, \beta$ with $\alpha<\beta$ be any two real numbers and let us denote the aggregate of points in $[a, b]$ such that $\alpha<f^{\prime}(x)<\beta$ by

$$
E_{\alpha \beta}=E\left(x ; \alpha<f^{\prime}(x)<\beta\right) ;
$$

then $E_{\alpha \beta}$ is either void or $\mathfrak{M}\left(E_{\alpha \beta}\right)>0$, where $\mathfrak{N}(E)$ is the Lebesgue measure of the set $E$.

2. In this note, we intend to give a more detailed description of $E_{\alpha \beta}$. We prove the following

THEOREM. The set $E_{\alpha \beta}$ gives rise to a set of nonoverlapping and nonabutting open sub-intervals $\left\{I_{i}\right\}$ in the space $[a, b]$ and a closed set $G$, which is the complementary set of $\left\{I_{i}\right\}$ with respect to $[a, b]$, such that $E_{\alpha \beta}$ is void in each $I_{i}$ and metrically dense everywhere in $G$.

3. We first show that $E_{\alpha \beta}$ is metrically dense in itself. For, take any point $P$ of $E_{\alpha \beta}$ and any neighborhood $U_{P}$ containing $P$ as its interior point, since $U_{P} \cap E_{\alpha \beta}$ is not void, from Clarkson's theorem, $\operatorname{Tr}\left(U_{P} \cap E_{\alpha \beta}\right)>0$. Thus, each point of $E_{\alpha \beta}$ is a limiting point of the set. I.e., $E_{\alpha \beta} \subseteq E_{\alpha \beta}^{\prime}$. Moreover, for each point $P \in C E_{\alpha \beta}^{\prime}$, it is always possible for us to construct a largest open sub-interval $I_{P}$ relative to the space $[a, b]$ such that it contains $P$ as its interior point and has its two end points belonging to the set $E_{\alpha \beta}^{\prime},{ }^{1}$ and such that all of its interior points are the points of $C E_{\alpha \beta}^{\prime}$. Thus, $E_{\alpha \beta}$ is void in each $I_{P}$, since $E_{\alpha \beta} \subseteq E_{\alpha \beta}^{\prime}$. Hence, we see that, corresponding to the set

${ }^{1}$ In case $a \in C E_{\alpha \beta}^{\prime}$ (similarly for $b$ ), the corresponding $U_{a}$ takes $a$ as one of its end points and the other end point of $U_{a}$ is of course a point of $E_{\alpha \beta}^{\prime}$. 
$C E_{\alpha \beta}^{\prime}$, there exists a set of open sub-intervals $\left\{I_{P}\right\}$, in each of which $E_{\alpha \beta}$ is void. Moreover, for any two different points $P$ and $P^{\prime}$ of $C E_{\alpha \beta}^{\prime}$, we have $I_{P} \equiv I_{P^{\prime}}$ or $I_{P} \cap I_{P^{\prime}}=0$. For, otherwise, there should be an interior point of $I_{P}$ (or $I_{P^{\prime}}$ ), which is also a point of the set $E_{\alpha \beta}^{\prime}$. This contradicts the mode of construction for $\left\{I_{P}\right\}$. Further, by the same reason, $I_{P}$ and $I_{P^{\prime}}$ can not be abutting. Therefore, the set of the open sub-intervals $\left\{I_{P}\right\}$ corresponding to the set $C E_{\alpha \beta}^{\prime}$ is a countable set of nonoverlapping and nonabutting open sub-intervals $\left\{I_{i}\right\}$ in the space $[a, b]$. Let the complementary set of $\left\{I_{i}\right\}$ with respect to $[a, b]$ be $G$. Then $G$ is closed. And $E_{\alpha \beta}$ is metrically dense everywhere in $G$, since, for any point $Q \in G, \operatorname{Tr}\left(U_{Q} \cap E_{\alpha \beta}\right)>0$ is satisfied for any arbitrary neighborhood of $Q$. This proves the theorem.

\section{REFERENCE}

1. J. A. Clarkson, A property of derivatives, Bull. Amer. Math. Soc. vol. 53 (1947) pp. 124-125.

National TAIWAN University

\section{A $q$-BINOMIAL COEFFICIENT SERIES TRANSFORM}

BY H. W. GOULD

Communicated by R. P. Boas, June 11, 1960

Following a standard notation $[1 ; 4 ; 5]$ we define the $q$-binomial coefficients by means of

$$
\left[\begin{array}{l}
x \\
n
\end{array}\right]=\prod_{j=1}^{n} \frac{q^{x-j+1}-1}{q^{j}-1},
$$

or sometimes more conveniently by the notation

$$
\left[\begin{array}{l}
x \\
n
\end{array}\right]=[x]_{n} /[n] !
$$

where

$$
\begin{gathered}
{[x]_{n}=[x][x-1] \cdots[x-n+1]} \\
{[x]=\left(q^{x}-1\right) /(q-1),} \\
{[n] !=[n]_{n}, \quad[0] !=[x]_{0}=1, \quad\left[\begin{array}{l}
x \\
0
\end{array}\right]=1 .}
\end{gathered}
$$

